Pacific Journal of Mathematics

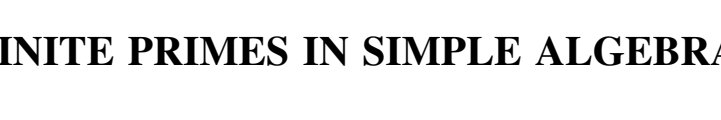




\title{
FINITE PRIMES IN SIMPLE ALGEBRAS
}

\author{
HOYT D. WARNER
}

A "prime" in an arbitrary ring with identity, as defined by $D$. K. Harrison, is shown to be a generalization of certain objects occurring in the classical arithmetic of a central simple $K$-algebra $\Sigma$, i.e., the theory of maximal orders over Dedekind domains with quotient field $K$. Specifically, if $K$ is a global field the "finite primes" of $\Sigma$ (in Harrison's sense) which contain a $K$-basis for $\Sigma$ are the generators of the Brandt Groupoids of normal $R$-lattices, $R$ ranging over the nontrivial valuation rings of $K$. The situation when $\Sigma$ contains a finite prime invariant under all $K$-automorphisms is studied closely; when $K$ is the rational numbers or $\operatorname{char}(K) \neq 0$, and $\sum$ has prime power degree, such a prime exists if and only if $\Sigma$ is a division algebra.

The techniques developed here are applied to yield new information concerning the generators and factorization in the Brandt Groupoids over certain Dedekind domains.

Harrison showed in [7] that his definition of prime (see $\S 1$ ) yields the finite and real infinite prime divisors of number fielas, as well as yielding the "primes" (i.e., prime ideals) of the rings of integers of these fields. His conjecture ([7, p. 13, footnote $]$ ), that a suitable modification of this definition existed which would yield objects in the arithmetic of "noncommutative number fields", was the starting point for this investigation; $\S 3$ contains the results.

Notation. Throughout this paper all algebras will be assumed finite dimensional and all fields considered will be assumed not locally finite unless explicitly stated otherwise. (By locally finite we mean each element is contained in a finite subfield.) $\boldsymbol{Z}, \boldsymbol{Q}$, and $\boldsymbol{R}$ will denote the integers, rationals, and reals, respectively. For sets $A$ and $B$,

$$
A \backslash B=\{x \in A \mid x \notin B\} .
$$

1. Preliminaries. In this section we prepare for the body of the paper by fixing notation and definitions we will need and proving some preliminary general results. We refer the reader to [7] for all unproved assertions about primes.

A preprime of a ring $R$ with identity 1 is a nonempty subset closed under addition and multiplication and not containing -1 ; a prime is a maximal preprime. A prime $P$ is called finite if $1 \notin P$, which is equivalent to $a \in P$ if and only if $-a \in P$, i.e., $P$ is an 
additive subgroup of $R$; otherwise $P$ is called infinite. If $P$ is finite, $A_{P}=\{a \in R \mid a P \subseteq P$ and $P a \subseteq P\}$ is a subring of $R$ and $A_{P} / P=k_{P}$ is a locally finite field. If $P$ is a finite prime of a field $F$, then $A_{P}$ is a valuation ring of $F$ with $P$ its ideal of nonunits. When $F$ is a global field the finite primes of $F$ are thus in one-to-one correspondence with the finite prime divisors of $F$. We note also that for $F$ a number field, the infinite primes which generate $F$ as a ring (called real infinite primes in [7]) are in one-to-one correspondence with the real infinite prime divisors (i.e., the real places) of $F$ [7, Proposition 3.5]. We will say a finite prime $P$ of a field $F$ is discrete rank one if the valuation determined by $A_{P}$ is nontrivial and discrete rank one. If $R \subseteq S$ are rings, $P$ and $T$ are primes of $R, S$, respectively, we shall say "T extends $P$ " if and only if $T \supseteqq P$, which is equivalent to $T \cap R=P$. Note that if $T$ extends $P$ then $T$ is finite if and only if $P$ is finite.

The following lemma generalizes [7, Proposition 2.1] to noncentral elements of a ring.

Lemma 1.1. Let $P$ be a finite prime of a ring $R$. Let $a, b \in R$. Then $a P b \subseteq P$ and $a b \in P$ imply $a \in P$ or $b \in P$.

Proof. Suppose $a P b \subseteq P, a b \in P$, but neither $a$ nor $b$ are in $P$. For $i \geqq 1$, let $W_{i}(a)$ be the set of all finite sums of elements of $R$ of the form $\alpha_{0} a \alpha_{1} a \cdots \alpha_{i-1} a \alpha_{i}$, where $\alpha_{j} \in Z \cdot 1+P$ for $0 \leqq j \leqq i$; let $W_{0}(a)=P$ and note $W_{i}(\alpha)$ is an additive group for each $i$. Let $T_{a}=\sum_{0}^{\infty} W_{i}(a)$; this is precisely the smallest additively and multiplicatively closed subset of $R$ containing $P$ and $a$, hence it must contain -1 since $a \in P$ implies $T_{a} \supsetneq P$, and $P$ is a maximal preprime. Likewise $-1 \in T_{b}$ (defined similarly). Write

$$
-1=\alpha+f_{1}(\alpha)+\cdots+f_{n}(\alpha)
$$

and

$$
-1=\beta+g_{1}(b)+\cdots+g_{m}(b)
$$

where $f_{i}(a) \in W_{\imath}(a), \quad g_{i}(b) \in W_{i}(b), \alpha, \beta$ are in $P$, and where $n$ and $m$ are chosen minimal $(n \geqq 1, m \geqq 1$ as $-1 \notin P)$. An easy induction argument shows that for $i \geqq j \geqq 1, W_{i}(a) W_{j}(b) \subseteq W_{i-j}(a)$ and for $1 \leqq i \leqq j, W_{i}(a) W_{j}(b) \subseteq W_{\jmath_{-i}}(b)$, because $a P b \leqq P$ and $a b \in P$ implies $a(Z \cdot 1+P) b \subseteq P$. If now $n \leqq m$, multiply (I) on the right by $-g_{m}(b)$ (after transposing $\alpha$ to the left side) which yields, by the preceeding remark, $(1+\alpha) g_{m}(b) \in \sum_{m-n}^{m-1} W_{j}(b)$. Then multiply (II) on the left by $1+\alpha$, transpose the $-\alpha$ to the right side and an expression like (II) results, but with the right side now in $\sum_{0}^{m-1} W_{i}(b)$, contradicting 
the minimality of $m$. If $n \geqq m$, the argument is similar.

The facts in the following corollary were first observed by Manis and Harrison (unpublished).

Corollary 1.2. Let $P$ be a finite prime of $R$.

(1) If $\mathfrak{p}$ is the largest two sided ideal of $R$ contained in $P$, then $\mathfrak{p}$ is a prime ideal.

(2) If $a, b \in$ center of $R, a b \in P$ implies $a \in P$ or $b \in P$, and $a b \in A_{P}, a \notin A_{P}$ imply $b \in P$.

(3) If $R_{1}$ is a subring of the center of $R$ and $P_{1}=P \cap R$ is a prime of $R_{1}$, then $A_{P} \cap R_{1}=A_{P_{1}}$.

(4) If $K$ is a subfield of the center of $R$ then $P \cap K$ is a prime of $K$, and $A_{P \cap K}=A_{P} \cap K$.

Proof. (1): If $\mathfrak{a} \cdot \mathfrak{b} \subseteq \mathfrak{p}, \mathfrak{a}$ and $\mathfrak{b}$ two sided ideals then $\mathfrak{a} P \mathfrak{b} \leqq$ $a R \mathfrak{b} \subset \mathfrak{p} \subseteq P$, so if $\mathfrak{a} \nsubseteq \mathfrak{p}$, i.e., $\mathfrak{a} \nsubseteq P$, the lemma implies $\mathfrak{b} \subseteq P$, i.e., $\mathfrak{b} \subseteq \mathfrak{p}$. (2): $a b \in A_{P}$ and $a, b \in$ center implies $a P b=a b P \subseteq P$ so $a \notin A_{P}$ implies $a \alpha \notin P$ for some $\alpha \in P$, but $\alpha \alpha P b \subseteq a P b \subseteq P$ and $(\alpha \alpha) b \in a P b \leqq$ $P$ then implies $b \in P$. (3): $A_{P} \cap R_{1} \subseteq A_{P_{1}}$ is clear. $P_{1} \subseteq P \subseteq A_{P}$, so suppose $a \in A_{P_{1}} \backslash P_{1}$; then as $A_{P_{1}} / P_{1}$ is a locally finite field, $a^{n}-1 \in P_{1}$ for some $n$; so $a^{n} \in 1+P_{1} \subseteq 1+P \subseteq A_{P}$, and hence $a \in A_{P}$ by (2). (4): By (2), $A_{P} \cap K$ is a valuation ring; since $\left(A_{P} \cap K\right) /(P \cap K)$ is a subring, hence a subfield, of the locally finite field $A_{P} / P, P \cap K$ is the maximal ideal of $A_{P} \cap K$ and is a prime of $K$ by [7, Proposition 2.5].

The following corollary shows that in studying the primes of a finite dimensional $K$-algebra $\Sigma$, it is sufficient to study the case when $\Sigma$ is simple, as we shall do in this paper.

Corollary 1.3. Let $\Sigma$ be a K-algebra, let $C$ be the center of $\Sigma$. If $T$ is any finite prime of $\Sigma, T \cap C$ is a finite prime of $C$. Every finite prime of $\Sigma$ contains the radical $\operatorname{rad} \Sigma$ of $\Sigma$ so there is a oneto-one correspondence between the primes of $\Sigma$ and those of the semisimple algebra $\bar{\Sigma}=\Sigma / \operatorname{rad} \Sigma$. The primes of a semi-simple algebra $\Pi_{1}^{n} \Sigma_{i}, \Sigma_{i}$ simple, are precisely those subsets of the form $T_{i} \cdot \prod_{j \neq i} \Sigma_{j}$ where $T_{i}$ is a prime of $\Sigma_{i}$.

Proof. Note that if $R$ is a ring, a a two sided ideal of $R$ then $P \supseteqq a$ is a preprime or prime of $R$ if and only if $P / a$ is a preprime or prime of $R / \mathrm{a}$. Next observe that if $P$ is a prime of $R$ and $R=$ $\mathfrak{b}_{1}+\cdots+\mathfrak{b}_{n}, \mathfrak{b}_{i}$ two sided ideals with $\mathfrak{b}_{i} \mathfrak{b}_{j} \subseteq P$ for $i \neq j$ then all but one of the $\mathfrak{b}_{i}$ is contained in $P$, by (1) of 1.2 . Hence $P /\left(\sum_{j \neq i} \mathfrak{b}_{j}\right)$ is a prime of the ring $R / \sum_{j \neq i} \mathfrak{b}_{j}$. The last two assertions now follow 
immediately, observing that as $\operatorname{rad} \Sigma$ is nilpotent, $\operatorname{rad} \Sigma \cong T$ for any finite prime $T$ by (1) of 1.2. That $T \cap C$ is a prime of $C$ follows from the preceeding remarks and (4) of 1.2.

In $\S 3$ and $\S 4$ below we shall be considering finite primes $T$ of a simple $K$-algebra $\Sigma$ which satisfy $K \cdot T=\Sigma$, i.e., such that $T$ contains a $K$-basis for $\Sigma$. The following proposition suggests that, by analogy with the commutative case, it is not unreasonable to impose this condition on (finite) primes of a noncommutative algebra.

Proposition 1.4. Let $\Sigma$ be a K-algebra.

(1) If $\Sigma$ is commutative then $K T=\Sigma$ for any finite prime $T$ of $\Sigma$.

(2) $\Sigma$ always contains finite primes $T$ with $K T=\Sigma$.

(3) Let $C$ be the center of $\Sigma$, let $L$ be any subfield of $C$ such that $\Sigma$ is finite dimensional over $L$. For a finite prime $T$ of $\Sigma$, the following are equivalent:

(a) $K T=\Sigma$

(b) $K C=\Sigma$

(c) $K L=\Sigma$.

Proof. First note that $T \cap K$, a prime of $K$ by 1.3 , is $\neq 0$ (as $K$ is not locally finite) so $K T \supseteqq A_{T}$ as $T \supseteqq(T \cap K) A_{T}$; thus $K T=\Sigma$ if and only if $K A_{T}=\Sigma$. Proof of (1): If $a \in \Sigma$ then $[\Sigma: K]<\infty$ implies $a^{n}+\alpha_{1} a^{n-1}+\cdots+\alpha_{n}=0$ for $\alpha_{i} \in K$, not all zero. $T \cap K=P$ is a finite prime of $K$ by 1.2 , (4), and so $A_{P}=A_{T} \cap K$ is a valuation ring of $K$ by [7, Proposition 2.5] and there exists $a \neq 0$ in $P$ with $\alpha \alpha_{i} \in P$ for $1 \leqq i \leqq n$. One checks that then $\alpha a$ is integral over $A_{P}$, so a fortiori over $A_{T}$, hence $\alpha a \in A_{T}$ as $A_{T}$ is integrally closed by [7, Proposition 2.7]. Thus $a \in K \cdot A_{T}$, and (1) holds. (2): If $\Sigma$ is arbitrary, let $P$ be a finite prime of $K ; P \neq\{0\}$ and $A_{P}$ is a valuation ring of $K$. If $1=x_{1}, x_{2}, \cdots, x_{n}$ is a $K$-basis of $\Sigma$ and $x_{i} x_{j}=\sum_{k} \gamma_{i j_{k}} x_{k}, \gamma_{i j k} \in K$ for $i, j \geqq 2$, we can choose $\alpha \neq 0$ in $P$ such that $\alpha \gamma_{i j k} \in A_{P}$ for all $i, j, k$ (using $A_{P}$ a valuation ring of $K$ ); then one checks $S=P+\sum_{2}^{n} A_{P}\left(\alpha x_{j}\right)$ is a preprime of $\Sigma$ containing $P$ and a $K$-basis for $\Sigma$; any prime $T$ containing $S$ is as desired. (3): By Corollary 1.3, $T \cap C$ is a (finite) prime of $C$, and hence by (1) above, (since $[C: K] \leqq[\Sigma: K]<\infty) K \cdot(T \cap C)=C ;[\Sigma: L]<\infty$ implies $L$ also is not locally finite so also $L \cdot(T \cap C)=C$. Hence $K \cdot T=C \cdot T=L \cdot T$, proving the assertion.

Definition 1.5. Let $\Sigma$ be a $K$-algebra (recall we assume $\Sigma$ finite dimensional, $K$ not locally finite). A finite prime $T$ of $\Sigma$ will be called spanning if $K \cdot T=\Sigma$. Proposition 1.4 shows this definition does not depend on the choice of the (not locally finite) field $K$ 
over which $\Sigma$ is finite dimensional and is equivalent to requiring $C \cdot T=\Sigma$ for $C$ the center of $\Sigma$.

We conclude this section by stating the main result of [10] and deriving a corollary we will need.

Proposition 1.6. ([10, Th. 3.2 and Corollary 2.2].) Let $k$ be a locally finite field, $\Sigma=\operatorname{Hom}_{k}(V, V)$ for $V$ a finite dimensional k-vector space. Every prime $T$ of $\Sigma$ is finite and has the form $T=$ $L(W, U)=\{f \in \Sigma \mid f(W) \subseteq U\}$ for uniquely determined k-subspaces $W \supseteq U$ of $V$ with $\operatorname{dim}_{k} W / U=1$ and conversely every such $L(W, U)$ (i.e., with $\operatorname{dim}_{l} W / U=1$ ) is a prime of $\Sigma$.

COROLlary 1.7. Situation as above, with $\operatorname{dim}_{k} V>1$. Every prime $T$ has the form $T=\varepsilon_{1} \Sigma+\Sigma \varepsilon_{2}$, where $1=\varepsilon_{1}+\varepsilon_{2}+\varepsilon_{3}$, the $\varepsilon_{i}$ are orthogonal idempotents, and $\varepsilon_{3}$ has rank $1\left(\right.$ so $\left.\varepsilon_{3} \neq 0\right)$.

Proof. $T=L(W, U), \quad \operatorname{dim}_{k} W / U=1$ so $W=k x \oplus U$ for some $x$; write $V=W^{\prime} \oplus W=U \oplus W^{\prime} \oplus k x$. Let $\varepsilon_{1}, \varepsilon_{2}, \varepsilon_{3}$ be the idempotents associated with the decomposition (i.e., $\varepsilon_{1}=$ projection on $U$ along $W^{\prime} \oplus k x$, etc.). Then $\varepsilon_{1} \Sigma=L(V, U) \subseteq L(W, U)$ and $\Sigma \varepsilon_{2} \leqq$ $L(W, 0) \subseteq L(W, U)$ so $T \subseteq \varepsilon_{1} \Sigma+\Sigma \varepsilon_{2}$. For the reverse inclusion, let $t \in T$. Then as $\left(1-\varepsilon_{2}\right) V=\left(\varepsilon_{1}+\varepsilon_{3}\right) V=U \oplus k x=W$ and as $t \cdot W \leqq$ $U \subseteq$ kernel of $1-\varepsilon_{1},\left(1-\varepsilon_{1}\right) t\left(1-\varepsilon_{2}\right)=0$ so $t\left(1-\varepsilon_{2}\right)-\varepsilon_{1} t\left(1-\varepsilon_{2}\right)=0$ so $t=\varepsilon_{1}\left(t\left(1-\varepsilon_{2}\right)\right)+t \varepsilon_{2} \in \varepsilon_{1} \Sigma+\Sigma \varepsilon_{2}$.

2. Brandt groupoid generators over classical dedekind domains. First we recall some facts and establish notation. Throughout this section $R$ will denote a Dedekind domain distinct from its quotient field $K$, and $\Sigma$ will denote a separable (see [4]) $K$-algebra. We refer the reader to [4], [5], and [6] for the following facts. An $R$-lattice $M$ in $\Sigma$ is a finite (i.e., finitely generated) $R$-submodule of $\Sigma$ with $K \cdot M=\Sigma$. An $R$-order in $\Sigma$ is a subring which is an $R$-lattice; every $R$-order is contained in a maximal one. If $M$ is an $R$-lattice, $O_{l}(M)=\{x \in \Sigma \mid x M \subseteq M\}$ and $O_{r}(M)=\{x \in \Sigma \mid M x \leqq M\}$ are $R$-orders, the left and right orders of $M . \quad M$ is called integral if $M \subseteq O_{l}(M)$ and normal if $O_{l}(M)$ is maximal; these definitions are really rightleft symmetric. If $A$ is an $R$-order then all nonzero prime (ring) ideals of $\Lambda$ are maximal ideals and are $R$-lattices. If $\Lambda$ is a maximal order the set of all $R$-lattices in $\Sigma$ which are two sided $\Lambda$-modules forms an abelian group free on the maximal ideals of $\Lambda$ as generators, with identity $\Lambda$ and inverses $M^{-1}=\{x \in \Sigma \mid M x M \cong M\}$; when $\Sigma$ is simple the set of all normal $R$-lattices forms a groupoid, called the Brandt Groupoid. Briefly, for $M, N$ normal $R$-lattices, the product 
$M \cdot N=\left\{\sum m_{i} n_{i} \mid m_{i} \in M, n_{i} \in N\right\}$ is allowed only when $O_{r}(M)=O_{l}(N)$; the maximal orders are the units; $N^{-1}=\{x \in \Sigma \mid N x N \leqq N\} ; N N^{-1}=$ $O_{l}(N), N^{-1} N=O_{r}(N)$; every normal $R$-lattice $M=N_{1} \cdot N_{2}^{-1}$ with $N_{1}$, $N_{2}$ integral, and every integral $R$-lattice $M$ has an essentially unique decomposition $M=N_{1} \cdots N_{k}$ (product in the groupoid) where the $N_{\imath}$ are indecomposable (i.e., not expresible as a groupoid product of nonunit integral normal lattices); the indecomposable $R$-lattices are exactly the maximal one-sided ideals in the maximal $R$-orders in $\Sigma$, and are called the generators of the Brandt Groupoid (over $R$ in $\Sigma$ ).

The main result of this section is a characterization of the generators of the Brandt Groupoid over $R$, when $R$ satisfies the additional condition that each maximal ideal $P$ of $R$ is a prime of $R$, or equivalently $R / P$ is a locally finite field for each maximal ideal $P$. We shall call such Dedekind domains "classical", since this condition is satisfied by all Dedekind domains whose quotient fields are (possibly infinite) algebraic number fields, and is also satisfied by the valuation rings of classical local fields; moreover, any Dedekind domain which is finitely generated (as a ring) is classical by [5, p. 68, Th. 3].

THEOREM 2.1. Let $R$ be a classical Dedekind domain, $\Sigma$ a simple separable algebra over the quotient field $K$ of $R$. The generators of the Brandt Groupoid over $R$ in $\Sigma$ are the maximal finite $R$-module preprimes of $\Sigma$. Moreover, each generator is a prime of any $R$-order containing it.

Proof. Let $I=I_{R}$ be the set of generators of the Brandt Groupoid over $R$ in $\Sigma$, and let $W=W_{R}$ be the set of all preprimes of $\Sigma$ which are also finite $R$-submodules of $\Sigma$. We are to prove that $I$ is the set of maximal elements of $W$.

If $S \in W$ then $R+S$ is a subring which is a finite $R$-module so is contained in an $R$-order $\Lambda$ (Proposition 1.1 of [4]) so $S \subseteq \Lambda$; thus the elements of $S$ are integral over $R$. A key step is the following:

Lemma 2.2. Let $S \in W$. Let $P$ be any maximal ideal of $R$ with $P \supseteqq S \cap R$. Let $\Lambda$ be any $R$-order containing $S$. Then there exists a positive integer $n$ with $P^{n} \Lambda+S \in W$, i.e., $P^{n} \Lambda+S$ is a preprime.

Proof. $1 \notin S$ so $S \cap R$ is a proper ideal of $R$ and $P$ exists. $P^{n} \Lambda+S \notin W$ for all positive integers $n$ means $1 \in P^{n} \Lambda+S$ for all $n$ (as $P^{n} \Lambda+S$ is a finite $R$-module closed under + and $\cdot$ ). Let $R_{P}$ be the localization of $R$ at $P$, let $M_{P}=R_{P} \cdot M$ for any $R$-submodule of $\Sigma$. Then $1 \in P_{P}^{n} \Lambda_{P}+S_{P}$ for all $n$ which implies, as $R_{P}$ is a Noetherian local ring and $\Lambda_{P}$ is a finite $R_{P}$-module, that $1 \in S_{P}$ 
(Proposition 6, of Chapt. 3, $\S 3$, no. 3 of [5]). But then there exists $s \in R \backslash P$ such that $s \cdot 1=s \in S$ i.e., $s \in S \cap R \cong P$, a contradiction.

Combining the initial remarks with the lemma above, any $S \in W$ is contained in $S_{1} \in W$ with $S_{1} \cdot K=\Sigma$. Now apply Zorn's lemma to the inductive collection of preprimes $T$ of $\Sigma$ such that $T \supseteqq S_{1}, T$ is an $R$-submodule of $\Sigma$ and $T$ consists of elements of $\Sigma$ integral over $R$; let $T$ be maximal such. Then $R+T$ is a ring of elements integral over $R$ and $K(R+T) \supseteqq K \cdot S_{1}=\Sigma$ so $R+T$ is an $R$-order of $\Sigma$ hence a finite $R$-module, hence $T \in W$, and clearly $T$ is maximal in $W$ (since we noted any $S \in W$ consists of integral elements). Thus any element $S$ of $W$ is contained in a maximal element $T$ of $W$.

Now suppose $T$ is any maximal element of $W . \quad T \cap R=P$ is a maximal ideal (as $T \cap R \subseteq P$, a maximal ideal of $R$, implies $T+P$ is a preprime and a finite $R$-module so $T=T+P$ ) and $P$ is a prime of $R$ as $R$ is classical. Next let $\Lambda$ be any $R$-order containing $T$, we claim $T$ is a prime of $\Lambda$. For suppose $T \subseteq T_{1}$ a prime of $\Lambda$. Then $T_{1} \cap R$ is a preprime of $R$ containing the prime $P=T \cap R$ of $R$, so $T_{1} \cap R=P$, and hence by Corollary 1.2, (3) $R=A_{P}=A_{T_{1}} \cap R$, i.e., $T_{1}$ is an $R$-module. But $T_{1} \subseteq \Lambda$ a finite $R$-module so $T_{1}$ is a finite $R$-module so $T_{1} \in W$ and $T_{1}=T$ by maximality of $T$ in $W$.

Now let $\Lambda$ be any maximal $R$-order containing $T$, a maximal element of $W$. We contend $T$ is either a left or a right ideal of $\Lambda$, hence a maximal left or right ideal of $\Lambda$ (as all proper ideals of $\Lambda$ are in $W$ ) and hence a generator of the Brandt Groupoid. By Lemma 2.2, $T$ contains a nonzero two-sided ideal of $A$ so by Corollary 1.2 , (1), the largest two-sided ideal $\mathfrak{p}$ of $\Lambda$ contained in $T$ is a nonzero prime ideal hence a maximal ideal of $\Lambda$. Hence (as $T$ is a prime of $A) \bar{T}=T / p$ is a prime of the simple $\bar{R}=R / P$ algebra $\bar{A}=$ $\Lambda / \mathfrak{p}$. But $\bar{R}$ is a locally finite field so any finite dimensional division algebra over $\bar{R}$ is again a locally finite field (see [7]), so either $\bar{A}$ is a locally finite field or $\bar{\Lambda} \cong \operatorname{Hom}_{k}(V, V), k$ locally finite, $2 \leqq$ $\operatorname{dim}_{k} V<\infty$. In the first case $\bar{T}=\{0\}$ i.e., $T=\mathfrak{p}$ and we are done (this is always the case when $\Sigma$ is commutative). In the second case, by Corollary 1.7, $\bar{T}=\varepsilon_{1} \bar{\Lambda}+\bar{\Lambda} \varepsilon_{2}$ with $1=\varepsilon_{1}+\varepsilon_{2}+\varepsilon_{3}$ in $\bar{\Lambda}, \varepsilon_{i}$ orthogonal idempotents in $\bar{\Lambda}$, and $\varepsilon_{3} \neq 0$. Choose $e_{i} \in \Lambda, 1 \leqq i \leqq 3$ with $e_{1}+\mathfrak{p}=\varepsilon_{1}, e_{2}+\mathfrak{p}=\varepsilon_{2}, e_{3}=1-\left(e_{1}+e_{2}\right)$, so $e_{3}+\mathfrak{p}=\varepsilon_{3}$ and $1=$ $e_{1}+e_{2}+e_{3}$. Then $T=e_{1} \Lambda+\Lambda e_{2}+\mathfrak{p}$. Our assertion about $T$ will be proven if we can show either $e_{1} \in \mathfrak{p}$ or $e_{2} \in \mathfrak{p}$ (as then $T=e_{1} \Lambda+\mathfrak{p}$, a right ideal, or $=\Lambda e_{2}+\mathfrak{p}$, a left ideal). So suppose both $e_{1}$ and $e_{2}$ are not in $\mathfrak{p}$. Let $S=T+e_{1} \mathfrak{p}^{-1} e_{2}$; using $e_{i} e_{j} \in \mathfrak{p}$ if $i \neq j \quad$ (as $\varepsilon_{i} \varepsilon_{j}=0$ if $i \neq j$ ) one checks that $S$ is closed under multiplication as well as addition. If $-1 \in S$, then $1 \in S$ (as $-S \subseteq S$ ) so as $-e_{1}-e_{2} \in T \subseteq S$, 
$e_{3}=1-e_{1}-e_{2} \in S$. But then $e_{3} e_{3} e_{3} \in e_{3} S e_{3}$ which one checks (using again $e_{i} e_{j} \in \mathfrak{p}$ if $i \neq j$ ) is contained in $\mathfrak{p}$, so $\varepsilon_{3}=\left(\varepsilon_{3}\right)^{3}=\left(e_{3}\right)^{3}+\mathfrak{p}=0$, a contradiction. Hence $-1 \notin S$, so $S \in W$ (as $S$ is clearly a finite $R$-module), and hence $S=T$ as $T$ is maximal in $W$. Then $e_{1} \mathfrak{p}^{-1} e_{2} \subseteq$ $S=T \subseteq \Lambda$, but as $\Lambda\left(e_{i}+\mathfrak{p}\right) \Lambda=\Lambda$ since $e_{i} \notin \mathfrak{p}$ for $i=1,2, e_{1} \mathfrak{p}^{-1} e_{2} \subseteq \Lambda$ implies $\mathfrak{p}^{-1}=\Lambda \mathfrak{p}^{-1} \Lambda=\Lambda\left(e_{1}+\mathfrak{p}\right) \Lambda \mathfrak{p}^{-1} \Lambda\left(e_{2}+\mathfrak{p}\right) \Lambda \subseteq \Lambda$, a contradiction.

To complete the proof of 2.1 it remains to show that any $T \in I$, i.e., any generator of the Brandt Groupoid, is maximal in $W$; but if $T$ were not maximal we would have $T \varsubsetneqq T_{1}$ maximal in $W$, but then $T_{1} \in I$ and (by Satz. 16, p. 76 of [6]) $T=M T_{1} N$ for $M, N$ normal integral $T$-lattices, $M$ or $N$ not a unit in the groupoid, contradicting $T$ indecomposable.

REMARKs. (1) If $\Sigma$ is a separable field extension then $I_{R}$ is the set of nonzero prime ideals of the integral closure $\bar{R}$ of $R$ in $\Sigma$, so the theorem characterizes them "intrinsically".

(2) The final assertion of the theorem shows that a maximal $R$-order $\Lambda$ in $\Sigma$ again is "classical" since the maximal one-sided ideals of $\Lambda$ are primes of $\Lambda$.

(3) The finite $R$-module preprimes $S$ with $K S=\Sigma$ are precisely the proper integral $R$-lattices ( $M$ proper meaning $M \varsubsetneqq 0_{l}(M)$ ), so the theorem shows every proper integral $R$-lattice is contained in a maximal one, which is in fact a normal lattice.

(4) It follows immediately from the proof above (or use [6]) that if $T$ is a generator of the Brandt Groupoid over $R$ in $\Sigma$, then $T$ is contained in exactly two maximal $R$-orders, namely $O_{l}(T)$ and $O_{r}(T)$.

3. Spanning primes of a simple algebra. In this section we apply Theorem 2.1 in the special case when $R$ is the valuation ring $A_{P}$ of a discrete rank one prime $P$ and $\Sigma$ is central, to show that the generators of the Brandt Groupoid of normal $A_{P}$-lattices are then precisely the spanning primes of $\Sigma$ extending $P$. With this we can characterize spanning primes over global fields. A close analysis of the case when $A_{P}$ is complete and use of Rutherford's Theorem (Proposition 1.6) yields some apparently new information about the factorization of the unique maximal ideal of a maximal $A_{P}$-order as a product of generators.

THEOREM 3.1. Let $\Sigma$ be a central simple K-algebra. Let $P$ be a discrete rank one finite prime of $K$. For $T \subseteq \Sigma$ the following are equivalent:

(1) $T$ is a spanning prime of $\Sigma$ extending $P$;

(2) $T$ is a prime of $\Sigma$ extending $P$ and $T$ is a finite $A_{P}$-module; 
(3) $T$ is a generator of the Brandt Groupoid of normal $A_{P^{-}}$ lattices in $\Sigma$.

Proof. We first assume that $A_{P}$ is a complete discrete rank one valuation ring and prove 3.1, then deduce the general case. The proof depends on the special case when $\Sigma$ is a division algebra, in which the situation is particularly simple.

Proposition 3.2. Let $D$ be a (not necessarily central) division algebra over $K$. Let $P$ be a finite prime of $K$ with $A_{P}$ a complete discrete rank one valuation ring. Then there is a unique prime $T$ of $D$ extending $P$. Namely, $T$ is the unique maximal one or twosided ideal of the unique maximal $A_{P}$-order $\Delta$ in $D . T$ is a spanning prime of $D$ and $T$ is a finite $A_{P}$-submodule of $D$.

Proof. Let $T$ be any prime of $D$ with $T \supseteqq P$; such a $T$ always exists by Zorn's lemma, and $T$ is a finite prime if $P$ is. Then $T \cap K=P$ and $A_{P} \subseteq A_{T}$ (by Corollary 1.2, (3)) so that $T$ is an $A_{P^{-}}$ submodule of $D$. To show $T=\mathfrak{p}$, the unique maximal one or twosided ideal of $\Delta$, it suffices to show $T \subseteq \Lambda$. For then $T$ is a finite $A_{P}$-module and so a maximal finite $A_{P}$-module preprime, hence by Theorem 2.1, $T=\mathfrak{p}$, as $\mathfrak{p}$ is the only maximal one-sided ideal of a maximal $A_{P}$-order in $D$. Let $t \in T$. If $t \in K$ then $t \in T \cap K=P \subseteq A_{P} \subseteq \Delta$. If $t \in T \backslash K$ let $f(x)=\sum_{0}^{n} a_{i} x^{i}$ be the monic minimum polynomial for $t$ over $K$, so $a_{n}=1$. Since $\Sigma$ is a division algebra and $t \neq 0, f$ is irreducible. Let $v$ be the (exponential) valuation determined by $A_{P}$. Then $v\left(a_{0}\right)>\min \left\{v\left(a_{i}\right) \mid 1 \leqq i \leqq n\right\}$, for if not, we have $v\left(a_{0}^{-1} a_{i}\right) \geqq 0$, $1 \leqq i \leqq n$, so $a_{0}^{-1} f(x)=a_{0}^{-1} a_{n} x^{n}+\cdots+a_{0}^{-1} a_{1} x+1$ has all its coefficients in $A_{P} \subseteq A_{T}$, but then $0=a_{0}^{-1} a_{n} t_{n}+\cdots+a_{0}^{-1} a_{1} t+1$ implies $-1=$ $a_{0}^{-1} a_{n} t^{n}+\cdots+a_{0}^{-1} a_{1} t \in A_{T} \cdot T \subseteq T$, a contradiction. Hensel's Lemma holds in $K$ so ([11, Lemma 21, p. 52]), $f(x)$ irreducible over $K$ implies $\min \left\{v\left(a_{i}\right) \mid 1 \leqq i \leqq n-1\right\} \geqq \min \left\{v\left(a_{0}\right), v\left(a_{n}\right)\right\}$. Now, $\min \left\{v\left(a_{0}\right), v\left(a_{n}\right)\right\}=$ $v\left(a_{n}\right)=v(1)=0$ by what we just showed, so $v\left(a_{i}\right) \geqq 0$ for all $i$ and $f(x) \in A_{P}[x]$, proving $t$ is integral over $A_{P}$, and hence $t \in \Delta$. Done.

Proof of 3.1 in the case that $A_{P}$ is complete, and $\Sigma$ is not a division algebra: applying Theorem 2.1, we see immediately that (2) implies (3) since (2) implies $T$ is a maximal element of the set of finite $A_{P}$-module preprimes in $\Sigma$; moreover, as $T$ is then a normal $R$-lattice, $K \cdot T=\Sigma$, so also (2) implies (1).

To show (1) implies (2) we identify $\Sigma$ with $(D)_{n}$ where $D$ is a division algebra finite dimensional (and central) over $K$, and $n>1$. Let $T$ satisfy (1) and let $\left\{\varepsilon_{i j} \mid 1 \leqq i, j \leqq n\right\}$ be the usual matrix units ( $\varepsilon_{i j}$ has 1 in $i, j$-th spot, 0 elsewhere). Let $\pi$ be a prime element of 
$A_{P}$, i.e., $\pi A_{P}=P$. Then $K T=\Sigma$ implies there exists a positive integer $k$ with $\pi^{k} \varepsilon_{i j} \in T$ for all $i, j$. We assert that $T \subseteq\left(\pi^{-2 k} T_{0}\right)_{n}$ where $T_{0}$ is the unique prime of $D$ extending $P$ (see 3.2 ); if so then since $T \cap K=P$ implies $T$ is an $A_{P}$-module (by (3) of Corollary 1.2) and since $T_{0}$ is a finite $A_{P}$-module (by Proposition 3.2), $T$ will be a finite $A_{P}$-module, proving (2) holds. Identify $D$ with the set of "scalar matrices", i.e., matrices of the form $\operatorname{diag}\{a, a, \cdots, a\}$ where $a \in D$. Since $D$ is a subring of $\Sigma, T \cap D$ is a preprime of $D$, and $\mathrm{T} \cap D$ extends $P$, so $T \cap D$ must be contained in $T_{0}$, the unique prime of $D$ extending $P$. To prove $T \subseteq\left(\pi^{-2 k} T_{0}\right)_{n}$ it then suffices to show $\pi^{2 k} M_{i j} \subseteq T \cap D$ where $M_{i j}=\{a \in D \mid a$ is the $i, j$-th entry of some matrix in $T\}$. Let $a \in M_{i j}$, say $a=a_{i j}$ for $t=\left(a_{l m}\right) \in T$. Then $\operatorname{diag}\left\{\pi^{2 k} a, \cdots, \pi^{2 k} a\right\}=\sum_{l=1}^{n}\left(\pi^{k} \varepsilon_{l i}\right) t\left(\pi^{k} \varepsilon_{j l}\right) \in T \cdot T \cdot T \cong T$ so $\pi^{2 k} a \in T \cap D$ as asserted.

Thus (1) is equivalent to (2). Finally if (3) holds, i.e., $T$ is a generator of the groupoid, let $T_{1}$ be any prime of $\Sigma$ containing $T$; then $K T_{1} \supseteqq K T=\Sigma$ so (1) holds for $T_{1}$, hence (2) holds for $T_{1}$, so $T_{1} \in W_{A P}$ so $T_{1}=T$ as $T$ is maximal in $W_{A_{P}}$ by Theorem 2.1. Thus (2) and (1) hold for $T$, concluding the proof of 3.1 when $A_{P}$ is complete.

To deduce the general case we apply the following well known lemma:

Lemma 3.3. Let $V$ be a finite dimensional K-vector space. Let $A$ be a discrete rank one valuation ring of $K$, with maximal ideal $P$. Let $A^{*}$ denote the P-adic completion of $A$.

(1) If $M$ is any A-submodule of $V,\left(M \otimes_{A} A^{*}\right) \cap V=M$.

(2) If $N^{*}$ is any $A^{*}$-lattice in $V \bigotimes_{A} A^{*}$, then $N^{*} \cap V=N$ is an A-lattice in $V$ and $N \otimes_{A} A^{*}=N^{*}$.

Proof. See Chapter 7 of [5]. One easily checks that $M$ need not be a finitely generated $A$-module in (1).

Proof of Theorem 3.1. Suppose first that $T$ satisfies (1) of Theorem 3.1. Then $T$ is an $A_{P}$-submodule of $\Sigma$ and $K T=\Sigma$. Hence (letting ( ) ${ }_{P}^{*}$ denote $P$-adic completion) $T^{*}=T \otimes A_{P}^{*}$ is an $A_{P}^{*}$-submodule of $\Sigma_{P}^{*}=\Sigma \otimes K_{P}^{*}, T^{*} \supseteqq P \cdot A_{P}^{*}=P^{*}$ the maximal ideal of $A_{P}^{*}$ (a prime of $K_{P}^{*}$ ) and $T^{*}$ spans $\Sigma_{P}^{*}$; moreover $T^{*}$ is a preprime of $\Sigma_{P}^{*}$ as $-1 \in T^{*}$ implies $-1 \in T^{*} \cap \Sigma=T$ (by 3.3, (1)), a contradiction. Now, letting $T_{1}$ be any prime of $\Sigma_{P}^{*}$ with $T_{1} \supseteqq T^{*}, T_{1}$ is a spanning prime extending $P^{*}$ and hence a finite $A_{P}^{*}$-module. This proves $T^{*}$ is an $A_{P}^{*}$-lattice in $\Sigma_{P}^{*}$, so $T=T^{*} \cap \Sigma$ is an $A_{P}$-lattice in $\Sigma$ by 3.3 , (2), and (2) of Theorem 3.1 holds. (2) immediately implies (3) by Theorem 
2.1. Finally, if (3) holds, suppose $T$ is not a prime, and let $T_{1}$ be any prime of $\Sigma$ containing $T$. Then $K T_{1} \supset K T=\Sigma$ (as $T$ is an $A_{P^{-}}$ lattice) so $T_{1}$ is a spanning prime and hence by what we just showed $T_{1}$ is a finite $A_{P}$-module. But $T$ is a maximal finite $A_{P}$-module preprime by Theorem 2.1 so $T=T_{1}$, a contradiction. Thus 3.1 is proven.

REMARK. If $\Sigma$ is a division algebra, (1)-(3) of Theorem 3.1 are equivalent to: $T$ is a prime of $\Sigma$ extending $P$ and $T$ consists of $A_{P^{-}}$ integral elements. For suppose this condition holds and let $K T=$ $\Sigma_{0} \subseteq \Sigma$. As $\Sigma$ is a division algebra, $\Sigma_{0}$ is a division algebra with center $L \supseteqq K$. Let $\mathrm{S}=T \cap L$, a prime of $L$ by Corollary 1.2, (4). $K T=\Sigma_{0}$ implies $T$ is a spanning prime of $\Sigma_{0}$ (by Proposition 1.4) so (as $A_{S}$ must again be a discrete rank one valuation ring) $T$ is a finite $A_{S}$-module by 3.1. As $S \subseteq T$ all elements of $S$ are $A_{P}$-integral so by [5, p. 151, Proposition 6], $A_{S}$ is the integral closure of $A_{P}$ in $L$. Therefore $A_{S}$ and hence $T$ is a finite $A_{P}$-module. But then $K \cdot T=\Sigma$ by 3.1 so (1) of 3.1 holds. Conversely if $T$ satisfies (1) hence (3) of 3.1 then $T$ is contained in an $A_{P}$-order of $\Sigma$ so the elements of $T$ are $A_{P}$-integral.

Theorem 3.1 essentially verifies Harrison's conjecture in $[7, p$. 13, footnote 3]. Explicitly:

THEOREM 3.4. Let $K$ be a field such that all its (nontrivial) valuations are discrete rank one, or equivalently such that all its finite primes are discrete rank one; for example, any global field. Let $\Sigma$ be a central simple $K$-algebra. Then the spanning finite primes of $\Sigma$ are the generators of the Brandt Groupoids over the nontrivial valuation rings of $\Sigma$. Specifically, if $T$ is spanning then $T \cap K=P$ is a finite prime of $K$ and $T$ is a generator of the Brandt Groupoid of normal $A_{P}$-lattices in $\Sigma$.

Proof. Immediate from Corollary 1.2 and Theorem 3.1.

Turning again to the situation of Theorem 3.1 we study the complete case more closely, with the aid of Rutherford's Theorem (Proposition 1.6), and obtain information on the groupoid generators, i.e., spanning primes in $\Sigma$, which divide the maximal ideal of a maximal order.

Theorem 3.5. Let $\Sigma$ be a central simple K-algebra. Let $P$ be a discrete rank one finite prime of $K$. Let $\Lambda$ be a maximal $A_{P}$-order of $\Sigma$ with unique maximal ideal $\mathfrak{p}$.

(1) The primes of $\Sigma$ which contain $\mathfrak{p}$ are precisely those generators of the Brandt Groupoid of normal $A_{P}$-lattices which divide, 
i.e., contain p.

(2) The map $T \rightarrow^{*}(T \cap \Lambda) / p$ gives one-to-one correspondence between the generators which divide $\mathfrak{p}$ and the set of finite primes of the finite dimensional simple $k_{P}=A_{F} / P$-algebra $\bar{\Lambda}=\Lambda / \mathfrak{p}$, which set is completely described by Rutherford's Theorem (Proposition 1.6).

(3) For each generator $T$ which divides $\mathfrak{p}$ there is an integer $r=r(T, \mathfrak{p}) \geqq 1$ such that $T$ can only appear as the $r$-th term in any factorization of $\mathfrak{p}$ as a product (in the groupoid) of generators.

Proof. (1) is an immediate consequence of Theorem 3.1, as $T \supseteqq \mathfrak{p}$ implies $K T \supseteqq K \mathfrak{p}=\Sigma$ and $T \cap K \supseteqq \mathfrak{p} \cap K=P$. To prove (2) and (3) it suffices to consider the case when $A_{P}$ is complete, for $M \rightarrow M \otimes A_{P}^{*}$ and $M^{*} \rightarrow M^{*} \cap \Sigma$ gives an isomorphism between the Brandt Groupoid in $\Sigma$ over $A_{P}$ and that in $\Sigma_{P}^{*}$ over $A_{P}^{*}$ (see $\S 11$ of Chapter 6 of [6]). If $\Sigma_{P}^{*}$ is a division algebra then $\mathfrak{p}$ is already a prime, $\bar{\Lambda}$ is a locally finite field and there is nothing to prove. Hence we assume $A_{P}$ is complete and we identify $\Sigma$ with $\operatorname{Hom}_{D}(V, V), D$ a central division algebra over $K, V$ a right $D$-vector space with $2 \leqq \operatorname{dim}_{D} V<\infty$. Let $\Delta$ be the unique maximal $A_{P}$-order in $D$ with unique maximal ideal $\pi \Delta=\Delta \pi$ (where $\pi$ is not necessarily in $K$ ); $\Delta$ is a (noncommutative) discrete rank one valuation ring of $D$ (cf. [11, Chap. 2]).

For $M$ and $N$ right $\Delta$-submodules of $V$ let

$$
L(M, N)=\{a \in \Sigma \mid a M \leqq N\} .
$$

Let $\Lambda$ be the fixed maximal $A_{P}$-order of $\Sigma$. Then $\Lambda=L(E, E) \cong$ $\operatorname{Hom}_{\Delta}(E, E)$ for a free $\Delta$-submodule $E$ of $V$ with $\operatorname{rank}_{\Delta} E=n=\operatorname{dim}_{D} V$, and the unique maximal ideal $\mathfrak{p}$ of $\Lambda$ i.e., the radical of $\Lambda$, equals $L(E, E \pi)$. Let $\Omega_{E}$ denote the set of all free, rank $n, \Delta$-submodules $F$ of $V$ such that $F \subseteq E$ but $F \nsubseteq E \pi$.

Claim. (a) every maximal $R$-order $\Lambda^{\prime}$ equals $L(F, F)$ for a unique $F \in \Omega_{E}$; (b) every prime $T$ of $\Sigma$ with $T \supseteqq \mathfrak{p}$ has the form $T=L\left(W_{T}, U_{T}\right)$ for a unique pair of free, rank $n, \Delta$-submodules of $E$, with $W_{T} \supseteqq U_{T} \supseteqq$ $E \pi$ and $\operatorname{rank}_{\lrcorner}\left(W_{T} / U_{T}\right)=1$.

Proof. (a) follows since $E D=V$ and $L(F, F)=L\left(F^{\prime}, F^{\prime}\right)$ if and only if $F^{\prime}=F \pi^{k}$ for some $k$. For $(b)$, let $O_{l}(T)=L(F, F)$ with $F \in \Omega_{E}$. Then $T \supseteqq \mathfrak{p}^{\prime}=L(F, F \pi)$, the radical of $O_{l}(T)$, and $T / \mathfrak{p}^{\prime}$ is a maximal left ideal of $O_{l}(T) / \mathfrak{p}^{\prime}=\operatorname{Hom}_{\bar{u}}(\bar{F}, \bar{F})$ where $\bar{F}=\bar{F} / F \pi$ is an $n$-dimensional $\bar{\Delta}=\Delta / \pi \Delta$ vector space. But then $T / \mathfrak{p}^{\prime}=\operatorname{Hom}_{\bar{u}}(\bar{F}, \bar{U})$ for a uniquely determined $(n-1)$-dimensional $\bar{\Delta}$-subspace of $\bar{F}$. Letting $U \supseteqq F \pi$ be the unique $\Delta$-submodule of $F$ with $U / F \pi=\bar{U}$, we see that $T \subseteq L(F, U)$; but $L(F, U)$ is a preprime (as $F \supseteqq U$ ) so $T=$ 
$L(F, U)$. Letting $W_{T}=F, \quad U_{T}=U$ we have $E \supseteqq W_{T} \supseteqq U_{T}$ and $\operatorname{rank}_{4} W_{T} / U_{T}=1$; it remains to show $U_{T} \supseteqq E \pi$. Now as $W_{T} \in \Omega_{E}$, there exists $x \in W_{T} \backslash E \pi$. By Nakayama's Lemma, $E$ has a free $\Delta$-basis $x=x_{1}, x_{2}, \cdots, x_{n}$; then $E \pi=\sum x_{i} \pi \Delta$. For each $i$ let $t_{i} \in L(E, E)$ be defined by $t_{i} x_{j}=\delta_{1 j} x_{i} \pi$ (Kronecker $\delta$ ). Then $t_{i} \in L(E, E \pi)=\mathfrak{p} \subseteq T$ so $x_{i} \pi=t_{i} x \in t_{i} W_{T} \subseteq U_{T}$ for $i=1, \cdots, n$ proving $E \pi \subseteq U_{T}$. Assertion (b) is proven.

Now, if $T \supseteqq p$ and $T=L\left(W_{T}, U_{T}\right)$ as in (b) above, let $\bar{W}_{T}=W_{T} / E \pi$, $\bar{U}_{T}=U_{T} / E \pi$. Then $(T \cap \Lambda) / \mathfrak{p}=\left\{\bar{a} \in \bar{\Lambda}=\Lambda / \mathfrak{p} \mid \bar{a} \bar{W}_{T} \subseteq \bar{U}_{T}\right\}$ which, by Rutherford's Theorem, is a prime of $\bar{\Lambda}=\operatorname{Hom}_{\bar{\Delta}}(\bar{E}, \bar{E}$ ) (where $\bar{E}=/ E \pi$ ) since $\bar{W}_{T} \subseteq \bar{U}_{T}$ are $\bar{\Lambda}$-subspaces with $\operatorname{dim}_{\bar{u}} \bar{W}_{T} / \bar{U}_{T}=1$. Conversely, by lifting back to $\Lambda$ and then extending to a prime of $\Sigma$, any prime of $\bar{\Lambda}$ has the form $(T \cap \Lambda) / \mathfrak{p}$ for some prime $T \supseteqq \mathfrak{p}$. Thus $T \rightarrow(T \cap \Lambda) / \mathfrak{p}$ is onto the primes of $\bar{\Lambda}$; that the map is one-to-one follows from the uniqueness part of Rutherford's Theorem and the uniqueness of the representation of $T$ as $L\left(W_{T}, U_{T}\right)$.

Thus (2) is proven. To prove (3), let $T \supseteqq \mathfrak{p}$ and suppose $T=$ $L\left(W_{T}, U_{T}\right)$ where $W_{T}, U_{T}$ are chosen as in the claim above. We claim $r=\operatorname{rank}_{\bar{\Delta}}\left(W_{T} / E \pi\right)$ has the property claimed in (3). First, note that $O_{r}(T)=L\left(W_{T}, W_{T}\right)$ and $O_{l}(T)=L\left(U_{T}, U_{T}\right)$. In any factorization $\mathfrak{p}=$ $T_{1} \cdots \cdot T_{n}, O_{r}\left(T_{i}\right)=O_{l}\left(T_{i+1}\right)$ so $W_{T_{i}}=U_{T_{i+1}}$ and the factorization corresponds to the chain $E \pi=U_{T_{1}} \varsubsetneqq W_{T_{1}}=U_{T_{2}} \varsubsetneqq \cdots \varsubsetneqq W_{T_{n-1}}=$ $U_{T_{n}} \varsubsetneqq W_{T_{n}}=E$, with $\operatorname{rank}_{\Delta}\left(W_{T_{i}} / E \pi\right)=i$ as $\operatorname{rank}_{\Delta} W_{T_{i}} / W_{T_{i-1}}=1$.

Corollary 3.6. Suppose $A_{P} / P$ is a finite field (which is always true for the classical fields). Then the maximal ideal $\mathfrak{p}$ of a maximal $A_{P}$-order $\Lambda$ is divisible by only finitely many generators of the Brandt Groupoid over $A_{P}$ (although there are in general (see §4) infinitely many distinct maximal $A_{P}$-orders in $\Sigma$.)

Proof. $\Lambda / \mathfrak{p}$ is a finite ring.

4. The Non-split case; self conjugate primes. In this section we study the special situation which arises when a discrete rank one prime $P$ of $K$ does not have infinitely many extensions to spanning primes of $\Sigma$, and give a characterization of division algebras of prime power degree.

THEOREM 4.1. Let $\Sigma$ be a central simple K-algebra, and $P$ a discrete rank one finite prime of $K$. Then the following four conditions are equivalent:

(1) $\Sigma$ contains only finitely many spanning primes extending $P$;

(2) there is a unique prime $T$ of $\Sigma$ extending $P$; 
(3) $\Sigma_{P}^{*}$ is a division algebra (where $\Sigma_{P}^{*}=\Sigma \otimes K_{P}^{*}$, the P-adic completion);

(4) there is a prime $T$ of $\Sigma$ extending $P$ which is "self-conjugate" in $\Sigma$, i.e., $a T a^{-1}=T$ for all units $a \in \Sigma$.

Moreover, the following two conditions are equivalent and are implied by the first four; they are equivalent to the first four if $K$ is a global field:

(5) every prime $T$ of $\Sigma$ extending $P$ is spanning;

(6) $\Sigma$ is a division algebra and $P$ has a unique extension to each subfield $L$ of $\Sigma$ containing $K$.

Remarks. In condition (5), "is spanning" can be replaced by "is a finite $A_{P}$-module" or by "consists of $A_{P}$-integral elements". (2), (3) and either one of these alternate versions of (5) are equivalent when $\Sigma$ is merely simple and separable, e.g., when $\Sigma$ is a separable field extension of $K$.

We will say $P$ is nonsplit in $\Sigma$ if any of (1)-(4) above hold.

Proof. By $\S 3$ there is a one-to-one correspondence between the spanning primes of $\Sigma$ extending $P$ and the spanning primes of the $P$-adic completion $\Sigma_{P}^{*}$ of $\Sigma$ which extend $P^{*}$ (the prime of $K_{P}^{*}$ ). Either there is exactly one maximal $A_{P}^{*}$-order in $\Sigma_{P}^{*}$ or there are infinitely many, according as $\Sigma_{P}^{*}$ is a division algebra or is not. With $3.1,3.2$ and the remark at the end of $\S 2$, this implies that $\Sigma_{P}^{*}$ contains exactly one prime, or contains infinitely many spanning primes according as it is a division algebra or not. Thus (1) is equivalent to (3). Clearly (2) implies (1); (3) implies (2) for if $T_{1}$ is any prime of $\Sigma$ extending $P$ then by Lemma $3.3(1), T_{1} \cdot A_{P}^{*}$ is a preprime of $\Sigma_{P}^{*}$ which extends $P^{*}$, so $T_{1} \cdot A_{P}^{*} \subseteq T^{*}$ the unique prime in $\Sigma_{P}^{*}$ extending $P^{*}$, hence $T^{*} \cap \Sigma \supseteqq T_{1}$ which implies (as $T^{*} \cap \Sigma$ is a preprime) $T_{1}=T^{*} \cap \Sigma$, the unique spanning prime of $\Sigma$ extending $P$. Thus (1), (2), and (3) are equivalent.

(2) clearly implies (4). Suppose (4) holds and let $T$ be self conjugate. Suppose that (3) does not hold, i.e., that $\Sigma_{P}^{*}$ is not a division algebra. Then there are infinitely many maximal $A_{P}$-orders in $\Sigma$ which are all conjugate under inner automorphisms of $\Sigma$ (by Proposition 3.5 of [4]) and hence (using remark at end of $\S 2$ and Th. 3.1) given any spanning prime it has infinitely many distinct conjugates. Thus $T$ cannot be spanning. But we show that it is: for let $\Sigma_{0}=K \cdot T=\left\{\sum \alpha_{i} t_{i} \mid \alpha_{i} \in K, t_{i} \in T\right\}$, the $K$-subalgebra generated by $T$ (as $T \cdot T \subseteq T$ ). We assert that $K \cdot T$ contains every unit of $\Sigma$. If so, we are done, as one checks $\Sigma$ has a basis consisting of units. Let $u$ be a unit in $\Sigma$ and suppose $u \notin K T$. Then $u \notin A_{T}$ (as $T \cap K=$ $P \neq 0$ implies $\left.A_{T} \leqq K T\right)$ so $u T=T u \nsubseteq T$ and there exists $t \in T$ with 
$u t \notin T$; but $u^{-1}(u t) \in T$ and $u^{-1} T u T=T \cdot t \leqq T$ so by Lemma 1.1, $u^{-1} \in T \subseteq K T$. But $K T$ is a $K$-subalgebra of $\Sigma$ so $u^{-1} \in K T$ implies $u \in K T$, a contradiction. This completes the proof of the equivalence of conditions (1) to (4).

(5) is equivalent to (6): if (5) holds then $\Sigma$ must be a division algebra, for otherwise there exists $a \in \Sigma$ with $a^{2}=0$ and then $P+K a$ is a preprime of $\Sigma$ containing $P$ which is not a finite $A_{P}$-module, so cannot be contained in a spanning prime. (5) is equivalent to the assertion that every preprime $T$ of $\Sigma$ containing $P$ is a finite $A_{P^{-}}$ module, hence in particular every prime of a subfield $L \supseteqq K$ of $\Sigma$ which extends $P$ must be a finite $A_{P}$-module. But this implies, by [5, p. 151, Proposition 6] that $P$ has a unique extension to each such $L$, and (6) holds. Now suppose (6) holds and suppose $T$ is a prime of $\Sigma$ extending $P$. Each $x \in T$ is in the unique extension of $P$ to the subfield $K(x)$ of $\Sigma$, and so $x$ is integral over $A_{P}$ by the result of [5] just quoted. Hence $T$ is spanning by the remark following Theorem 3.3.

Clearly (2) implies (5) so any one of the first four conditions implies the last two. We conclude by showing that if $K$ is a global field (i.e., an algebraic number field or an algebraic function field over a finite field) then (6) implies (3), so that all the conditions are equivalent. Suppose then (6) holds and suppose that $\Sigma_{P}^{*}$ is not a division algebra. ${ }^{1}$ Let $n$ be the degree of $\Sigma\left(n^{2}=[\Sigma: K]\right)$. We have $\Sigma_{P}^{*} \cong(D)_{k}, k>1$ and degree $D=m<n$, with $m \mid n$. Let $P=$ $P_{0}, P_{1}, \cdots, P_{s}$ be a finite set of finite primes of $K$ including all the finite primes $Q$ at which $\Sigma$ is not unramified, i.e., all those $Q$ with $\Sigma_{Q}^{*}$ not split. By the Grunwald-Wang Theorem [3, p. 106, Th. 5] there exists a normal field extension $L$ of $K$ (which is even a cyclic extension) with the following properties (letting $n_{P}=\left[L_{S}^{*}: K_{P}^{*}\right]$ for $S$ any extension of $P$ to $L): n_{P}=m, n_{P_{i}}=n$ for $i=1, \cdots, s, n_{Q}=2$ for each real infinite prime $Q$ of $K$ for which $\Sigma_{Q}^{*}$ is not split, and $[L: K]=n$. Then by construction, for every finite or real infinite prime $Q$ of $K$, the $Q$-index of $\Sigma$ (i.e., the index of $\Sigma_{Q}^{*}$ ) divides the $Q$-degree of $L$ over $K$. Thus the $Q$-index of $\Sigma \otimes_{K} L$ is 1 for all $Q$ and $\Sigma \bigotimes_{K} L \cong(L)_{n}$ by Hasse's theorem ([12, p. 206, Th. 2]). But then by $[6$, p. 46 , Satz 14] and the fact that $[L: K]=n=$ degree of $\Sigma, L$ is isomorphic to a maximal subfield of $\Sigma$. Therefore, $P$ has a unique extension to a prime of $L$, which implies the $P$-degree of $L$ over $K$ must be $n$ (as the $P$-degree $=\mathrm{e} \cdot \mathrm{f}$ which equals $n$ as $e f g=n$, $g=1$ ) a contradiction to our construction of $L$ with $P$-degree $=m<n$.

REMark. The equivalence of (2) and (5) when $K$ is a global field shows that in general there exist nonspanning primes in $\Sigma$, see $\S 6$.

\footnotetext{
1 The following argument was suggested by M. Schacher.
} 
Proposition 4.2. Let $\Sigma, K$ and $P$ be as in Theorem 4.1 and suppose $P$ is nonsplit in $\Sigma$. Let $T$ be the unique prime of $\Sigma$ extending $P$. Then $A_{T}$ is the unique maximal $A_{P}$-order in the division algebra $\Sigma, T$ is its unique maximal ideal (one or two sided). The Brandt Groupoid over $A_{P}$ in $\Sigma$ is the cyclic group consisting of all powers of T. Moreover, $A_{T}$ is a (noncommutative) discrete rank one valuation ring of $\Sigma$ (see [11]), with $T$ its ideal of nonunits. $k_{T}=$ $A_{T} / T$ is a finite field extension of $k_{P}=A_{P} / P$, the value group $\Gamma_{T}$ of the valuation associated with $A_{T}$ is a finite extension of the value group $\Gamma_{P}$ of the $A_{P}$ valuation, and $\left[\Gamma_{T}: \Gamma_{P}\right] \cdot\left[k_{T}: k_{P}\right]=[\Sigma: K]$ i.e., "ef $=n ")$.

Proof. It suffices to prove the assertions of the last two sentences, the rest being immediate consequences of previous results. As $x A_{T} x^{-1}=A_{T}$ for all $x \neq 0$ in $A_{T}$, to show $A_{T}$ is a valuation ring we need only show $x \notin A_{T}$ implies $x^{-1} \in A_{T}$. But $x \notin A_{T}$ implies (as $x T=$ $T x$ ) that $x T \nsubseteq_{j}^{1} T$, say $x t \notin T$ for $\mathrm{t} \in T$. Then $x^{-1} x t \in T$ and $x^{-1} T x t \leqq T$ so by Lemma 1.1, $x t \notin T$ implies $x^{-1} \in T$, as required. The associated valuation is discrete rank one since $T$ is a principal ideal of $A_{T}$ by Corollary to Proposition 3.3 of [4]. By hypothesis $\Sigma_{P}^{*}$ is a division algebgra. $\quad T^{*}=T \cdot A_{P}^{*}$ is the prime of $\Sigma_{P}^{*}$ extending $P^{*}=P \cdot A_{P}^{*}$ and $A_{T}^{*}$ is a (noncommutative) valuation ring of $\sum_{P}^{*}$ with $\left[\Gamma_{T^{*}}: \Gamma_{P^{*}}\right] \cdot\left[k_{T^{*}}: k_{P^{*}}\right]=$ [ $\left.\Sigma_{P}^{*}: K_{P}^{*}\right]$ ([11, p. 54, Th. 11]). But $A_{T^{*}} / T^{*} \cong A_{T} / T$ (see [6]) and $A_{P^{*}} / P^{*} \cong A_{P} / P$, and also $P \cdot A_{T}=T^{e}$ and $P^{*} \cdot A_{T^{*}}=\left(T^{*}\right)^{e}$ so by order theory (see [6]) $\left[\Gamma_{T^{*}}: \Gamma_{P^{*}}\right]=e=\left[\Gamma_{T}: \Gamma_{\Gamma}\right]$, proving the last assertion.

We conclude this section by showing that over a global field the existence of a self conjugate finite or infinite prime characterizes the division algebras among all central simple algebras of prime power degree. Indeed when char $(K) \neq 0$ and $K$ has only discrete rank one valuations, Theorem 4.1 shows this result is equivalent, for algebras of prime degree, to the Hasse local splitting theorem (i.e., " $\Sigma$ is a matrix algebra if and only if $\Sigma_{P}^{*}$ is a matrix algebra for all primes $P$ of $K$ "). This suggests proving the Hasse splitting theorem for, say, (generalized) quaternion algebras over an arbitrary field of nonzero characteristic whose valuations are discrete rank one by directly proving the existence of a self-conjugate prime in such algebras. The author is indebted to D. K. Harrison for these observations and for conjecturing the following result.

Proposition 4.3. Let $K$ be a global field. Let $\Sigma$ be a central simple K-algebra of degree $p^{k}$ where $p$ is a prime number. If $K=Q$ or char $(K) \neq 0$, then $\Sigma$ is a division algebra if and only if there exists a self-conjugate prime in $\Sigma$. 
More generally, if $K$ is any global field then $\Sigma$ is a division algebra if and only if $\Sigma$ contains a self conjugate prime $T$, finite or infinite.

Proof. First suppose $\Sigma$ is a division algebra. The degree of $\Sigma=$ exponent of $\Sigma$ (in the Brauer Group of $K$ ) = the least common multiple of the $P$-exponents $m_{p}$ of $\Sigma$, i.e., of the exponents of the completions $\Sigma_{P}^{*}$ of $\Sigma$ at the primes $P$ (finite and real infinite) of $K$. But all the $P$-exponents are divisors of $p^{k}$, hence powers of $p$, and so for some $P$, the $P$-exponent of $\Sigma$ equals $p^{k}$, i.e., the index of $\Sigma_{P}^{*}$ is $p^{k}$. Since $p^{k}$ is the degree of $\Sigma_{P}^{*}, \Sigma_{P}^{*}$ is a division algebra. If $P$ is a finite prime we are done; when char $(K)=p \neq 0$ this must happen. So suppose $\operatorname{char}(K)=0$ and $P$ is real infinite. If $K=\boldsymbol{Q}$, so that $P$ is the unique infinite prime of $\boldsymbol{Q}$, then $\Sigma_{P}^{*}=H$ the ordinary (Hamiltonian) quaternions, so degree $\Sigma=2$, and since by Hasse Reciprocity $\sum_{\text {all } P}(\Sigma \mid P) \equiv 0(\bmod 1)$ (where $(\Sigma \mid P)$ is the Hasse invariant of $\Sigma$ at $P)$, there must exist a finite prime $P^{\prime}$ with $(\Sigma \mid P) \not \equiv 0(\bmod 1)$, hence with $m_{P^{\prime}}>1$, hence with $m_{P^{\prime}}=2$ (as $m_{P^{\prime}} \mid$ degree $\Sigma$ ) hence with $\Sigma_{P^{\prime}}^{*}$ a division algebra. Finally suppose $K$ is any algebraic number field. We have $\Sigma_{P}^{*}$ a division algebra, i.e., $\Sigma_{P}^{*}=H$. Then degree $\Sigma=2, \Sigma$ is a (generalized) quaternion division algebra over $K$. We assert that $P$ is in fact an (infinite) prime of $\Sigma$ and hence (as $P \subseteq K$ ) a selfconjugate prime of $\Sigma$ as required. Suppose $P \varsubsetneqq T$ a prime of $\Sigma$. Then (as $P$ is a prime of $K$ ) $T \nsubseteq K$, so let $a \in T \backslash K$. The subfield $K(a)$ of $\Sigma$ is a quadratic extension of $K$, in fact $K(a)=K(b)$ for some $b \in \Sigma$ with $b^{2} \in K$. Let $T_{0}$ be any infinite prime of $K(b)$ containing $a$ and extending $P\left(T_{0}\right.$ exists as $a$ and $P$ are in the preprime $T \cap K(b))$. By [7], $T_{0}$ is an archimedean order in the subfield $L$ of $K(b)$ which it generates; but (as $P$ is real infinite so is the cone of an ordering of $K$-see [7]) $L \supseteqq K$ and (as $\left.a \in T_{0}\right) \quad a \in L$ so $L=K(a)=$ $K(b)$. Hence $T_{0}$ is an order in $K(b)$ so $b^{2} \in T_{0}$, hence in $T_{0} \cap K=P$. But now consider $\sum_{P}^{*}=H$, and identify $K$ with a subfield of $\boldsymbol{R}$ via the unique isomorphism $K \rightarrow T$ which sends $P$ into $\boldsymbol{R}^{+}$, the nonnegative reals. Then $b \in H$ and $b^{2}=r \in P \subseteq \boldsymbol{R}^{+}$, and $r \neq 0$. Hence $r=t^{2}$ for some real number $t$ and we have $(b-r)(b+r)=0$ with $b-r$ and $b+r \neq 0$ as $b$ is not in the center $K$ of $\Sigma$, so not in the center $\boldsymbol{R}$ of $H$, which is a contradiction since $H$ is a division algebra.

Now suppose $\Sigma$ is any central simple algebra over the global field $K$ of arbitrary degree, and suppose $T$ is a self-conjugate prime of $\Sigma$. We show $\Sigma$ must be a division algebra. If $T$ is finite then we are done, by Theorem 4.1 (for condition (4) of 4.1 then holds with $P=K \cap T)$. Hence suppose $T$ is infinite and suppose $\Sigma \cong(D)_{n}$ with $n>1, D$ a central $K$-division algebra. First, if $T \subseteq K$ then for any 
$a \in \Sigma$ with $a^{2}=0, T+K a$ is a preprime properly containing $T$, a contradiction. Thus $T \nsubseteq K$. Let $T^{+}=\{a \in T \mid-a \notin T\}$. Then by Proposition 1.6 of [7], $G=T^{+} \cap U(\Sigma)$ is a multiplicative subgroup of $U(\Sigma)$, the units of $\Sigma$, which is $G L(n, D)$. Moreover, $G$ is a normal subgroup of $G L(n, D)$ since $a T a^{-1}=T$ implies $a T^{+} a^{-1}=T^{+}$for all $a \in U(\Sigma) . \quad G$ is not contained in the center $K^{\times}$(multiplicative group of $K$ ) (for let $a \in T \backslash K$, and choose $n$ a positive integer such that $-n$ is not an eigenvalue of the matrix $a \in(D)_{n}$, then $a+n \cdot I \in G \backslash K$.) Hence by Theorem 4.9 of [2], $G \supseteqq S L(n, D)$ the unimodular group and hence for all $a \in U(\Sigma), a \in G$ if and only if $\operatorname{det} a \in \operatorname{det} G=$ $\{\operatorname{det} b \mid b \in G\} \subseteq D^{\times} /\left(D^{\times}\right)^{\prime}$ (commutator factor group of $\left.D^{\times}\right)$. If $n$ is even we are done immediately, for then $\operatorname{det}(I)=\operatorname{det}((-1) \cdot I)(I=$ identity of $\Sigma$ ) so $-I \in G \subseteq T^{+} \subseteq T$ a contradiction. So suppose $n$ is odd. Then $\operatorname{det}(I)=\operatorname{det}(\operatorname{diag}\{1, \cdots, 1\})=\operatorname{det}(\operatorname{diag}-1, \cdots,-1,1\})$ (since $(-1)^{n-1}=1$ if $n$ is odd) and hence diag $\{-1, \cdots,-1,1\} \in G \subseteq T^{+}$. Then $\operatorname{diag}\{0, \cdots, 0,1\}$, which is the matrix unit $\varepsilon_{n n}$, is in $T^{+}+T^{+} \subseteq$ $T^{+}$. As $T^{+}$is self-conjugate this implies $\varepsilon_{i i} \in T^{+}$for $i=1, \cdots, n$. Finally, as for $i \neq j$, $\operatorname{det}\left(I+\varepsilon_{i j}\right)=1=\operatorname{det}\left(I-\varepsilon_{i j}\right)$, both $I+\varepsilon_{i j}$ and $I-\varepsilon_{i j}$ are in $T^{+}$, hence (multiply by $\varepsilon_{i i}$ on left, $\varepsilon_{j j}$ on right) both $\varepsilon_{i j}$ and $-\varepsilon_{i j}$ are in $T^{+}$, contradicting the definition of $T^{+}$. The proof is complete.

5. Miscellaneous results. In this brief section we prove the analogue, for a central simple algebra $\Sigma$ over a global field $K$, of the fact that each unit (i.e., nonzero element) of $K$ is contained in only finitely many primes of the field, and we discuss the topology on the space of all finite primes of $\Sigma$.

Let $X_{S}(\Sigma, P)=X_{S}(P)$ be the set of spanning primes of $\Sigma$ which extends the finite prime $P$ of $K . \quad X_{S}(P) \neq \varnothing$ by Proposition 1.4.

Proposition 5.1. Let $\Sigma$ be a central simple K-algebra, let $P$ be a discrete rank one finite prime of $K$. Any two elements of $X_{S}(P)$ are conjugate under a K-automorphism of $\Sigma$. If $X_{S}(P)$ is infinite, then

$$
P=\bigcap\left\{T \mid T \in X_{S}(P)\right\}, A_{P}=\bigcap\left\{A_{T} \mid T \in X_{S}(P)\right\} .
$$

Proof. The first assertion follows from Theorem 3.1, [2, Proposition 3.5], and the fact that maximal left ideals in a fixed maximal order are conjugate. The second assertion follows from the corresponding assertion about $X_{S}\left(\Sigma_{P}^{*}, P^{*}\right)$ ( $P$-adic completions), which holds as $\sum_{P}^{*}$ is not a division algebra by Theorem 4.1 .

Proposition 5.2. Let $K$ be a global field, $\Sigma$ a central simple 
$K$-algebra. For $a \in \Sigma$, a is an element of some $T \in X_{S}(\Sigma, P)$ for at most finitely many primes $P$ of $K$ if and only if $a$ is a unit in $\Sigma$.

Proof. Let $f(x)=x^{n}+\alpha_{1} x^{n-1}+\cdots+\alpha_{n}$ be the minimum polynomial for $a$ over $K ; a$ is a unit of $\Sigma$ if and only if $\alpha_{n} \neq 0$. The set $\left\{\alpha_{1}, \cdots, \alpha_{n}\right\} \subseteq A_{P}$ for all but a finite number of finite primes $P$ of $K$, as $K$ is a global field. If $\left\{\alpha_{1}, \cdots, \alpha_{n}\right\} \subseteq A_{P}$ then $a \in T$ for a spanning prime $T$ of $\Sigma$ extending $P$ if and only if $\alpha_{n} \in P$. For, $a \in T$ implies $\alpha_{n}=-a^{n}-\alpha_{1} a^{n-1}-\cdots-\alpha_{n-1} a \in A_{P} \cdot T \subseteq T$ so $\alpha_{n} \in T \cap K=$ $P$, while conversely if $\alpha_{n} \in P, a \cdot A_{P}[a]$ is a finitely generated $A_{P}$-module (since $a$ is integral over $A_{P}$ by hypothesis) and a preprime (as it is a proper ideal); therefore by $\S 2$ and $\S 4, a \cdot A_{P}[a]$, and hence $a$, is contained in a spanning prime extending $P$. Thus $a$ can be in some $T \in X_{S}(\Sigma, P)$ for infinitely many $P$ if and only if $\alpha_{n} \in P$ infinitely many $P$ if and only if $\alpha_{n}=0$.

We recall the topology on the space $Y(\Sigma)$ of all primes of $\Sigma$, as defined in [7]. A subbase for the open sets consists of the sets $W(a)=\{T \in Y(\Sigma) \mid a \notin T\}$ for all $a \in \Sigma$. The topology is $T_{1}$ and is not Hausdorff (in general $Y(R)$ is Hausdorff if and only if $R$ is a generalized Boolean ring: for all $a \in R$ there exists $n>1$ with $\left.a^{n}=a\right) . \quad Y(\Sigma)$ and the subspace $X(\Sigma)$ of all finite primes are both (quasi-) compact. A nonempty subset $U$ of a topological space $X$ is called irreducible if and only if $U$ is not the union of two nonempty closed proper subsets (or, every nonempty open subset is dense)

Proposition 5.3. Let $X_{S}(\Sigma)$ denote the space of spanning finite primes of the central simple K-algebra $\Sigma, K$ a global fie'd. Then $\bigcap\left\{T \mid T \in X_{S}(\Sigma)\right\}=\{0\} . \quad X_{S}(\Sigma)$ is a dense irreducible subset of $X(\Sigma)$ and $Y(\Sigma)$; thus the latter spaces are irreducible.

Proof. The first assertion follows from Proposition 5.1, the observation that $\Sigma_{P}^{*}$ is a division algebra for at most finitely many $P$, and the corresponding fact for $K$. For the second assertion it suffices to show: if $E=\left\{a_{1}, \cdots, a_{n}\right\}$ is any finite set of nonzero elements of $\Sigma$ then there exists $T \in X_{S}(\Sigma)$ with $T \cap E=\varnothing$. Let $f_{i}(x)$ be the minimal $K$-polynomial for $\alpha_{i}$, with constant term $\alpha_{i}$. If all the $\alpha_{i} \neq 0$ then choosing $P \in X(K)$ with $f_{i}(x) \in A_{P}[x]$ for all $i$ but with $\alpha_{i} \notin P$ for all $i$ we have $T \cap E=\varnothing$ for any spanning prime $T$ of $\Sigma$ extending $P$, by the argument of the proof of 5.2. In particular this proves the assertion when $\Sigma$ is a division algebra. If some $\alpha_{i}=0$ then $\Sigma \cong(D)_{n}, n>1$. Since the $a_{i}$ are nonzero one can choose a matrix representation for $\Sigma$ over $D$ in which the matrices for the $a_{i}$ all have nonzero last columns. Let $E_{0}=\{\delta \in D \mid \delta \neq 0, \delta$ is a lastcolumn entry in the matrix of some $\left.a_{i}\right\}$. By the first argument, there exists a spanning prime $T_{0}$ of $D$ with $T_{0} \cap E_{0}=\varnothing$. Let $T$ be 
the set of matrices with last column entries in $T_{0}$, all other entries in $\Lambda_{0}=O_{l}\left(T_{0}\right)$. One checks $T$ is a maximal left ideal of the maximal order $\left(\Lambda_{0}\right)_{n}$ in $(D)_{n} \cong \Sigma$, hence $T$ is a spanning prime of $\Sigma$ by 4.1 . By construction, $T \cap E=\varnothing$, and we are done.

6. Examples. In this section we give some examples of finite primes in simple algebras which are not spanning primes of the algebra.

The first set of example arises from an important description of finite primes containing idempotents, due to M. E. Manis (unpublished).

Proposition 6.1. Let $R$ be a ring, let $e$ be an idempotent of $R$, let $f=1-e$.

(a) Let $P=e P e$ be a finite prime of the ring eRe, with unit element e. A finite prime of $R$ may be constructed as follows: let $M_{0}$ be any additive subgroup of eRf; let $N=\left\{x \in f R e \mid M_{0} x \leqq e P e=P\right\}$, let $M=\{x \in e R f \mid x N \sqsubseteq P\}$ and finally let $B=\{x \in f R f \mid x N \leqq N\}$. Then $T=B+N+M+P=f B f+f N e+e M f+e P e$ is a finite prime of $R$. Note that $f \in T, e \notin T$.

(b) If $T$ is any prime of $R$ with $f \in T$, then $e T e=P$ is a finite prime of eRe; $T$ can be recovered from $P$ and, say, $M_{0}=e T f$ by the construction in $(a)$.

\section{Proof. Straightforward checking.}

Manis showed that in case $R$ is a full matrix ring over a locally finite field $k$ the above construction, with $e$ any idempotent of rank one, yields all the finite primes of $R$, giving an approach to the description of the primes of $R$ differing from that in [10].

Applying Proposition 6.1 to the construction of nonspanning primes in a matrix algebra $\Sigma=(D)_{n}, n>1$ over a division ring $D$, let $S$ be a finite prime of the center $K$ of $D$, let $P$ be any prime of $D$ extending $S$. Let $\left\{\mathrm{e}_{i j} \mid 1 \leqq i, j \leqq n\right\}$ be a set of matrix units in $\Sigma$. In the notation of 6.1 , let $e=e_{n n}$, let $M_{0}=\{0\}$. Then $N=f \Sigma e$, $M=\{0\}, B=f \Sigma f$. One checks that $T$ is the set of matrices with arbitrary entries in the first $n-1$ rows, and 0 entries in all but the $n$-th column of the $n$-th row, the $(n, n)$ entries being in $P$. Clearly $T$ does not span $\Sigma$ over $K$.

The preceding construction yields nonspanning primes in $\Sigma$ whether or not $D$ contains nonspanning primes. In case $D$ is a noncommutative central $K$-division algebra for $K$ an algebraic number field, the existence of nonspanning primes in $D$ follows from Theorem 4.1, or more directly from the fact that for any subfield $L$ of $D$ properly containing $K$, there are finite primes $P$ of $K$ which split in $L$. For to say $P$ splits in $L$ is to say that the primes $S_{1}, \cdots, S_{k}$ of 
$L$ extending $P$ are not integral over $A_{P}$, so that if $T$ is any prime of $\Sigma$ extending $S_{1}$, say, $T$ cannot be integral over $A_{P}$, hence (by 4.1 ) $T$ cannot be a spanning prime.

We now give a final example which shows that a prime of a central simple algebra may span a "small" subalgebra. Let $K$ be any (nonlocally finite) field, $L$ a cyclic Galois extension of $K$ with group $G=\langle\sigma\rangle$ of order $n$. Let $\Sigma=(L, \sigma, a)$ be a cyclic algebra $\left(\Sigma=\sum \bigoplus_{i=0}^{n-1} L u^{i}\right.$ with $u^{i} \alpha=\sigma^{i}(\alpha) u^{i}$ for $\alpha \in L$ and $u^{n}=\alpha \in K$-see [1] or [4]). Suppose $P$ is a finite prime of $K$ which splits completely in $L$, i.e., $P$ has $n$ distinct extensions $T_{1}, \cdots, T_{n}$ to a prime of $L$. Then each $T_{i}$ is a prime of $\Sigma$, with $K \cdot T_{i}=L,[L: K]=n=[\Sigma: K]^{1 / 2}$. Such primes exist in any algebra $\Sigma$ central simple over a global field $K$, as $\Sigma$ is then a cyclic algebra (see [1] or [6]), say $\Sigma=(L, \sigma, a)$ for some cyclic extension $L$ of $K$, and it can be shown (e.g., using the zeta function) that there exist in fact infinitely many finite primes of $K$ which split completely in $L$.

The author wishes to thank Professor Harrison who directed the thesis, from part of which this paper was developed. His enthusiasm and imagination were invaluable and the germs of many ideas herein were his.

\section{BIBLIOGRAPHY}

1. A. A. Albert, Structure of Algebras, A. M. S. Colloq. Pub. 24, Amer. Math. Soc., Providence 1961.

2. E. Artin, Geometric Algebra, New York, 1957.

3. E. Artin and J. Tate, Classified Theory, Benjamin, New York, 1967.

4. M. Auslander and O. Goldman, Maximal orders, Trans. Amer. Math. Soc. 97 (1960) $1-24$.

5. N. Bourbaki, Algebre Commutative, Chaps. 5 et 6 A. S. I. 1308, Hermann, Paris, 1964.

6. M. Deuring, Algebren, Springer Verlag, Berlin, 1968.

7. D. K. Harrison, Finite and infinite primes for rings and fields, Memoirs Amer. Math. Soc. 68 (1966).

8. N. Jacobson, Theory of Rings, Amer. Math. Soc. Survey 11, A. M. S. Providence, 1943.

9. - Structure of Rings, Amer. Math. Soc. Colloq. Pub. 37, Amer. Math. Soc. Providence, 1956.

10. H. Rutherford, Characterizing primes in some non commutative rings, Pacific J. Math. 27 (1968), 387-392.

11. O. F. G. Schilling, The Theory of Valuations, Amer. Math. Soc. Survey 4, Amer. Math. Soc. New York, 1950.

12. A. Weil, Basic Number Theory, Springer-Verlag, New York, 1967.

Received August 11, 1969. Supported by N. S. F. Grant \#GP-5340 and by a postdoctoral fellowship at the University of Illinois. 



\title{
PACIFIC JOURNAL OF MATHEMATICS
}

\author{
EDITORS
}

H. SAMELSON

Stanford University

Stanford, California 94305

C. R. Новву

University of Washington

Seattle, Washington 98105
J. DUGUNDJI

Department of Mathematics

University of Southern California

Los Angeles, California 90007

RICHARD ARENS

University of California

Los Angeles, California 90024

\section{ASSOCIATE EDITORS}

E. F. BECKENBACH

B. H. NeUManN

F. WOLF

K. YosHidA

\section{SUPPORTING INSTITUTIONS}

UNIVERSITY OF BRITISH COLUMBIA CALIFORNIA INSTITUTE OF TECHNOLOGY UNIVERSITY OF CALIFORNIA MONTANA STATE UNIVERSITY UNIVERSITY OF NEVADA NEW MEXICO STATE UNIVERSITY OREGON STATE UNIVERSITY UNIVERSITY OF OREGON OSAKA UNIVERSITY UNIVERSITY OF SOUTHERN CALIFORNIA
STANFORD UNIVERSITY UNIVERSITY OF TOKYO UNIVERSITY OF UTAH WASHINGTON STATE UNIVERSITY UNIVERSITY OF WASHINGTON

AMERICAN MATHEMATICAL SOCIETY CHEVRON RESEARCH CORPORATION TRW SYSTEMS

NAVAL WEAPONS CENTER 


\section{Pacific Journal of Mathematics}

\section{Vol. 36, No. $1 \quad$ November, 1971}

Norman Larrabee Alling, Analytic and harmonic obstruction on

nonorientable Klein surfaces ............................ 1

Shimshon A. Amitsur, Embeddings in matrix rings .............. 21

William Louis Armacost, The Frobenius reciprocity theorem and essentially bounded induced representations ....................... 31

Kenneth Paul Baclawski and Kenneth Kapp, Topisms and induced

non-associative systems ............................ 45

George M. Bergman, The index of a group in a semigroup ............ 55

Simeon M. Berman, Excursions above high levels for stationary Gaussian

processes....................................... 63

Peter Southcott Bullen, A criterion for $n$-convexity .............. 81

W. Homer Carlisle, III, Residual finiteness of finitely generated commutative

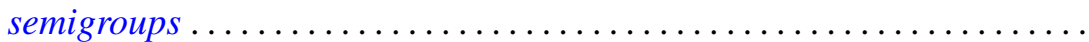

Roger Clement Crocker, On the sum of a prime and of two powers of

two ............................................ 103

David Eisenbud and Phillip Alan Griffith, The structure of serial rings . . . 109

Timothy V. Fossum, Characters and orthogonality in Frobenius

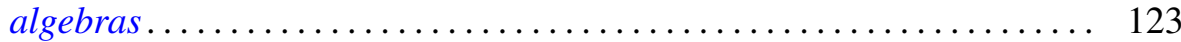

Hugh Gordon, Rings of functions determined by zero-sets . .......... 133

William Ray Hare, Jr. and John Willis Kenelly, Characterizations of Radon partitions...

Philip Hartman, On third order, nonlinear, singular boundary value

problems

David Michael Henry, Conditions for countable bases in spaces of

countable and point-countable type ..

James R. Holub, Hilbertian operators and reflexive tensor products ...

Robert P. Kaufman, Lacunary series and probability ..... . .

195

Erwin Kreyszig, On Bergman operators for partial differential equations in

two variables ................................

Chin-pi Lu, Local rings with noetherian filtrations . .

Louis Edward Narens, A nonstandard proof of the Jordan curve theorem...

S. P. Philipp, Victor Lenard Shapiro and William Hall Sills, The Abel summability of conjugate multiple Fourier-Stieltjes integrals. .

Joseph Earl Valentine and Stanley G. Wayment, Wilson angles in linear normed spaces

Hoyt D. Warner, Finite primes in simple algebras ...

Horst Günter Zimmer, An elementary proof of the Riemann hypothesis for an elliptic curve over a finite field... 